\title{
A função da nomeação oral sobre comportamentos emergentes de leitura e escrita ensinados por computador
}

\author{
José Gonçalves Medeiros \\ Analu Regis Fernandes \\ Raquel Pimentel \\ Ana Carolina Seara Simone \\ Universidade Federal de Santa Catarina
}

\begin{abstract}
Resumo
Os métodos de alfabetização utilizados em sala de aula não têm diminuído os índices de repetência nas séries iniciais. Por essa razão, o presente trabalho enfocou o comportamento de leitura e escrita como objeto de estudo. Para isso, utilizou-se o programa Mestre@ (Goyos \& Almeida, 1994). Os objetivos foram o ensino de um repertório de leitura e escrita, composto de palavras regularmente utilizadas nas séries iniciais, e o desenvolvimento e sistematização de um procedimento de ensino, via software. O procedimento utilizado foi o de exclusão. Foi solicitada a um grupo de alunos, a nomeação oral do estímulo modelo antes de escolher e/ou montar as palavras que estavam sendo ensinadas. Os resultados indicam uma alta probabilidade da aprendizagem sem erro das relações ensinadas bem como leitura das palavras de generalização, além de mostrar efeitos diferenciais entre os grupos, isto é, facilitação para o grupo sem o procedimento de nomeação oral.
\end{abstract}

Palavras-chave: equivalência de estímulos; alfabetização por computador; nomeação oral

\begin{abstract}
The oral naming function on emergencies behaviors of reading and spelling teaching by computer. The literate methods used in classroom haven't decreased the indexes of repetition in the initial grades. On that score, the present study focused on the behavior of reading and spelling. For this, the program Mestre@ (Goyos \& Almeida, 1994) was used. The goals were the teaching of a repertoire of reading and spelling, made up of words regularly used in the initial grades, and the development and systematization of a teaching procedure, through software. The procedure used was the match-to-sample. The oral naming of the sample stimulus by a group of students was required, before they could choose the words that were being taught. The results indicate a high probability of the learning without error of the teaching relations as well as of the reading of the generalization words. In addition, the findings show differential effects among the groups, that is to say, facilitation to the group without the oral naming procedure.
\end{abstract}

Key words: stimulus equivalence; computational literate; oral naming

A s escolas da rede pública de ensino apresentam um número elevado de crianças com dificuldades de aprendizagem. Uma prática comum, observada principalmente entre os professores, consiste em transferir para os alunos a culpa pelo fracasso, o que tem elevado os índices de repetência e evasão escolar, contribuindo, assim, para o aumento do fracasso escolar. Para uma melhor compreensão desse processo, foi desenvolvido um projeto em uma escola da rede pública, utilizando um programa de ensino informatizado desenvolvido por Goyos e Almeida (1996), com o objetivo de: (a) ensinar um repertório de leitura e escrita, composto de palavras comumente utilizadas nas séries iniciais; e (b) desenvolver e sistematizar um procedimento de ensino, via software, a grupos de crianças do ensino fundamental, em que um dos grupos seria ensinado através do uso de um procedimento de nomeação oral.

A abordagem utilizada deriva de um conjunto integrado de métodos desenvolvidos a partir de pesquisas na área de equivalência de estímulos (Stromer, Mackay, \& Stoddard, 1992). Quando o estímulo inclui palavras, equivalência pode 
ser livremente definida como aprendizagem daquilo que as palavras significam. Outras formas de comportamento, como escrever e soletrar palavras, podem também estar envolvidas. Resultados promissores têm sido obtidos, principalmente com crianças em idade escolar (de Rose, 1988; Medeiros, 1997; Medeiros, Antonakopoulu, Amorim, \& Righetto, 1997; Medeiros et al., 1995; Medeiros \& Silva, 2002; Melchiori, Souza, \& de Rose, 1992), mas também com adultos analfabetos (Medeiros, Monteiro, \& Zaccaron, 1997; Melchiori, Souza, de Rose, \& Furlan, 1992), com uma criança hiperativa com déficit de atenção (Medeiros \& Monteiro, 1996), e com crianças com paralisia cerebral (Rodrigues \& Medeiros, 2001). Em geral, independente da condição do participante (criança, adulto ou excepcional), os procedimentos têm garantido o ensino e promovido alterações socialmente desejáveis de relações comportamentais já estabelecidas, além de propiciar o surgimento de novas relações, denominadas de emergentes ou de generalização (de Rose, Souza, Rossito, \& de Rose, 1989; Hanna et al., 1993; Medeiros \& Teixeira, 2000; Stromer \& Mackay, 1992). Uma dificuldade encontrada em sujeitos deficientes mentais ou com dificuldade de aprendizagem é sua incapacidade de extrapolar para além dos limites do que lhes foi ensinado. Desse modo, os estudos de equivalência de estímulos oferecem um paradigma a ser explorado no ensino, especialmente no ensino de indivíduos que apresentam dificuldades de aprender pelos métodos convencionais.

Já foram relatados estudos aplicando este paradigma ao ensino do conceito de número (Monteiro \& Medeiros, 2002), ao ensino de habilidades de leitura e escrita a deficientes mentais (Mackay, 1985; Mackay \& Sidman, 1984) e ao ensino de habilidades de uso de moedas em sujeitos severamente retardados (McDonagh, McIlvane, \& Stoddard-Lawrence, 1984). Stromer (1991), em seu artigo "Equivalência de estímulos: implicações para o ensino", relata a revisão que Singh e Singh (1986) realizaram em relação às pesquisas sobre ensino de leitura para indivíduos com dificuldades especiais nas quais, segundo eles, os procedimentos baseados na equivalência de Sidman foram "exemplares em termos de sofisticação metodológica e provêm a melhor evidência de que habilidades básicas de leitura podem ser ensinadas para esta população" (p.190).

Com base nesse referencial teórico e, com o auxílio de um software educativo, foi desenvolvido e sistematizado um procedimento para se trabalhar no laboratório de informática da própria escola, com crianças que apresentavam dificuldades de aprendizagem. Podem ser encontradas duas posições sobre a forma mais adequada de utilização do computador em educação (Ribeiro, 1997). Uma delas afirma que deve ser usado somente como um recurso de aprendizagem, em que dispondo do instrumento necessário, o educando dirige sua própria aprendizagem. A outra posição afirma que o computador pode ser utilizado como um instrumento didático, fornecendo ao educando programas educacionais estruturados que buscam cumprir um determinado objetivo. As duas posições podem se complementar, pois o uso do computador como instrumento de ensino traz a vantagem de possibilitar a introdução de qualquer área do currículo nos diferentes momentos do processo educativo.
O computador pode operar em conjunto com outros recursos, facilitando a aprendizagem e enriquecendo-a pela motivação que possibilita (Gatti, 1993). Segundo a autora, altera-se a rotina da escola e a do professor quanto ao domínio e manipulação de informação e conhecimento, pois exige criatividade e flexibilidade. Ainda, segundo Ribeiro (1997), o recurso da informática, embora possa trazer algumas dificuldades, apresenta grandes vantagens desde que, em primeiro lugar, ocorra questionamento por parte do educador acerca de sua visão de homem e do processo de ensino que fará uso para o alcance de seus objetivos. O emprego do computador deveria, desse modo, favorecer a renovação do ensino e se constituir para o professor e para o aluno num evento problematizador da relação, dinamizando a reflexão sobre a prática e a metodologia pedagógica.

Neste sentido, o software Mestre@ (Goyos \& Almeida,1996) apresenta-se como um software promissor, pois se constitui num programa aberto, onde o professor pode inserir programas de qualquer natureza, com recursos para inserção de figuras e sons. É bastante amigável na linguagem computacional pela facilidade de manipulação e pela presença de um menu bem elaborado.

O trabalho que está sendo apresentado está relacionado teórica e metodologicamente àqueles que foram realizados nos últimos cinco anos, nos quais Medeiros, Konescki e Souza (1993) e Medeiros, Teixeira, Cabral, Silva e Brandão (1995) trabalharam com crianças com história de fracasso escolar em um programa de ensino de discriminações condicionais. Os modelos eram palavras faladas e os estímulos de comparação eram palavras impressas (compostas por duas e por três sílabas). Os resultados obtidos com esses projetos mostram um aumento significativo do número de palavras que as crianças passaram a ler em relação ao repertório de entrada, além da emergência de novas relações que surgiram a partir das relações condicionais ensinadas.

Ao excluir a palavra conhecida e escolher a nova diminui-se a probabilidade de insucesso na aquisição de novas palavras. Daí a denominação de aprendizagem sem erro. Para uma criança com história de fracasso ocasionada, em parte, por seus insucessos no processo de aquisição do repertório textual, um procedimento desta natureza deveria motivá-la (os estudos da área têm demonstrado) a prosseguir na tarefa escolar - o que possivelmente refletirá nas atividades da sala de aula.

Alguns estudos têm apontado que, além da cópia, a nomeação oral teria um efeito importante e diferenciador sobre a aquisição da leitura, generalização e na formação das classes de equivalência. Alguns autores apontam que um dos possíveis fatores de facilitação na emergência dessas relações seria a nomeação dos estímulos, nomeação essa com função mediadora (Devany, Hayes, \& Nelson, 1986; Dugdale \& Lowe, 1990; Hayes, 1989; Monteiro \& Medeiros, 2002), enquanto outros apresentam evidências empíricas contrárias a essa interpretação (Sidman, 1986, 1990, 1994; Sidman, Rauzin, Lazar, Cunningham, Tailby, \& Carrigan, 1982; Sidman, Wilson-Morris, \& Kirk, 1986). Numa série de estudos realizados e publicados em Temas de Psicologia, Matos, Peres, Hübner e Malheiros 
(1997) mostram que o treino de nomeação (fluente ou escandido), por si só, não produziu leitura recombinativa (generalizada), assim como o treino em cópia sem nomeação também não o fez. Para os autores, a rede deve ser ampliada com o treino simultâneo das habilidades adicionais (cópia e nomeação). Apontam, ainda, a importância da proximidade temporal da resposta de nomeação com a cópia na facilitação da transferência de controle de estímulos entre elementos do conjunto visual (C) e elementos do conjunto auditivo (A), transferência essa facilitada pela manipulação de sílabas escritas na atividade de montagem das palavras durante a cópia por construção.

Com base nos estudos descritos, o presente artigo relata os resultados de um procedimento de equivalência de estímulos computadorizado, com a utilização da nomeação oral com um dos grupos de crianças.

\section{Método}

\section{Participantes}

Participaram desse procedimento 11 crianças (6 do sexo masculino e 5 do feminino), agora denominados participantes (Ps ou P), que apresentavam dificuldades relacionadas à leitura e escrita de palavras, com idade variando entre 6 e 8 anos. Na época, no ano letivo de 2002, eram alunos da $1^{\underline{a}}$ e $2^{\underline{a}}$ séries, provenientes de duas turmas e professoras diferentes, de uma escola da rede pública de Florianópolis, Santa Catarina.

\section{Local}

O procedimento experimental foi realizado numa sala que funciona como o laboratório de informática da escola, com cortinas e janelas que dão visão para um pátio externo e para uma avenida com tráfego intenso. Nele estão instalados 15 computadores para uso de todos os alunos matriculados, sendo que em três deles foi instalado o software Mestre@ para o ensino de leitura e escrita com os Ps do presente procedimento.

\section{Material e equipamento}

Foram utilizados três computadores, com recurso multimídia, placa de som, alto-falantes Labtec LCS 150, CDROM, impressora e o software Mestre@ (Goyos \& Almeida, 1996). Fones de ouvido também foram utilizados para evitar a interferência de estímulos auditivos provenientes dos outros programas que estavam funcionando simultaneamente.

\section{Procedimento}

O procedimento principal foi o de exclusão, usado para expandir gradualmente, ao longo de uma seqüência de passos, o repertório de pareamentos entre modelos e estímulos de comparação. A base do procedimento de exclusão consiste na presença, em cada tentativa de pareamento, de um estímulo de comparação conhecido pelo participante, que é apresentado juntamente com outro estímulo de comparação desconhecido. Quando o experimentador apresenta o estímulo modelo correspondente ao estímulo de comparação desconhecido, o estímulo de comparação conhecido funciona como pista (ou deixa) para que o participante o rejeite e selecione corretamente o estímulo de comparação desconhecido. Assim, neste programa, o procedimento de exclusão permitiu uma alta probabilidade de aprendizagem sem erro das relações $\mathrm{AB}, \mathrm{AC}, \mathrm{AF}$ e $\mathrm{CF}$. O programa também envolveu a inserção de pré/pós-testes e teste extensivo de leitura e escrita como pode ser visto na Tabela 1.

O pré-teste verificou respectivamente a aquisição de leitura das palavras ensinadas na exclusão anterior (agora denominadas palavras de linha de base) e a leitura das palavras novas a serem ensinadas através do procedimento de exclusão (daqui por diante referidas como palavras de ensino), bem como de palavras novas não diretamente ensinadas (referidas como palavras de generalização), formadas a partir das sílabas das palavras de ensino da linha de base imediatamente anterior. O pós-teste avaliou as palavras ensinadas (rAC) na exclusão, bem como as palavras de generalização ( $\mathrm{rACg}$ ).

Ao término de uma exclusão e após ter atingido o critério para a realização da próxima exclusão, os Ps foram submetidos a: (1) um teste de ditado das palavras de ensino (rAE), cuja instrução foi "escrever a palavra ditada numa folha em branco"; (2) cópia de palavras de ensino impressas com conseqüenciação positiva, cuja instrução foi "escrever a palavra impressa num caderno de caligrafia” ( $\mathrm{rCF}$ ); e (3) repetição do teste de ditado como descrito em 1 (rAE), logo acima. $\mathrm{Na}$ análise dos dados referentes ao testes (como descrito em 1 e 3), os acertos foram classificados dentro de classes de 0$33 \%, 50-67 \%$ e $90-100 \%$. Assim, numa palavra dissílaba, o acerto de uma sílaba levava o pesquisador a classificar aquela palavra na classe de $50-67 \%$ de acertos. Numa palavra trissílaba, com o acerto de uma sílaba, o pesquisador classificava a palavra na classe de $0-33 \%$. O acerto de duas sílabas levava o pesquisador a classificar a palavra na classe de 50$67 \%$ de acertos, e assim por diante.

Testes para verificar a formação de equivalência entre os estímulos palavra impressa, palavra ditada e figura $(\mathrm{rBC}$ e rCB) foram conduzidos, além de testes de nomeação das palavras de ensino (rCDt), das palavras de generalização $(\mathrm{rCDg})$, das figuras (rBD) e montagem do nome das figuras (rBF). Em relação às palavras de generalização, foi realizado também um teste de montagem (anagrama), tendo como modelo o estímulo auditivo, para verificar se, além da nomeação correta, os Ps montavam corretamente as palavras de generalização (rAFg).

A Tabela 1 apresenta, à esquerda, um esquema do fluxo do programa de ensino e de testes e, à direita, indicado pela letra $x$, o número da exclusão alcançada e, por sombreamento, o número da exclusão não atingida. O programa foi dividido em uma seqüência de passos. Os dois primeiros passos foram destinados à aquisição de uma linha de base inicial, que foi posteriormente expandida com o emprego do procedimento de exclusão. O programa prosseguiu alternando passos de exclusão com passos de equivalência (numerados seqüencialmente), nos quais foi verificada a formação de classes de equivalência entre palavras faladas, palavras impressas e figuras. Ao término do programa foi realizado o teste final. 
Tabela 1

Fluxo da seqüência de passos e a exclusão (alcançada pelos participantes e a não realizada)

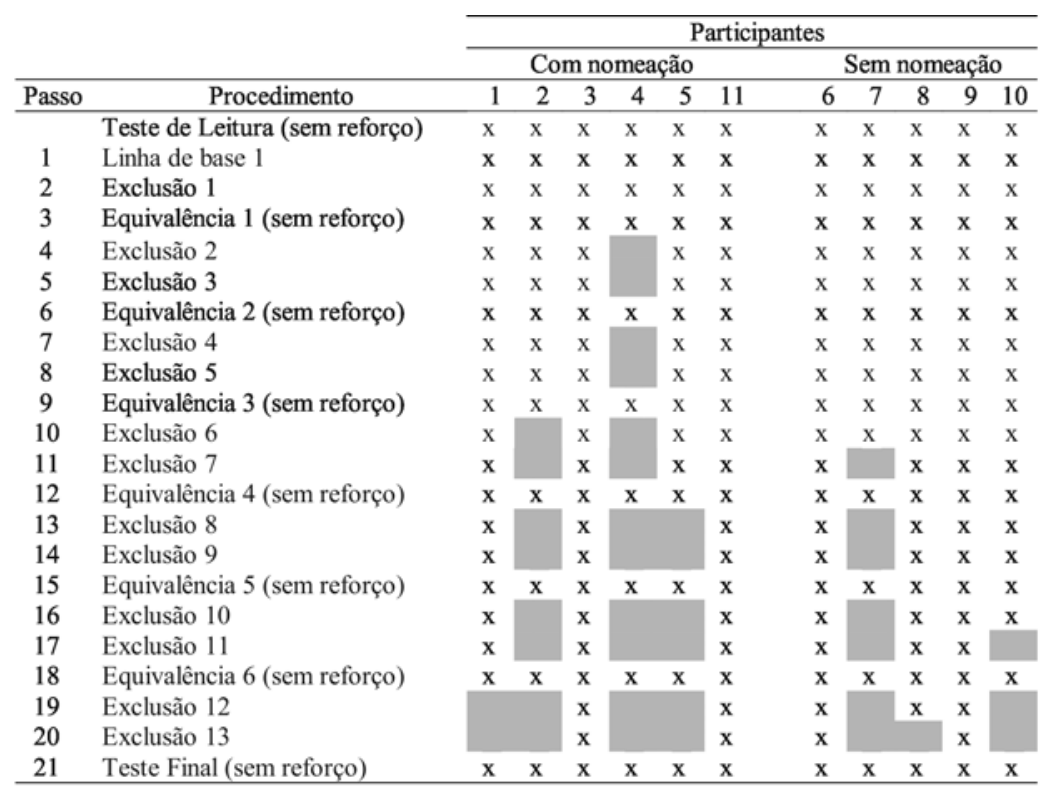

Nota: À esquerda, o fluxo da sequiência de passos do programa. Cada tipo de passo está numerado de acordo com sua ordem de inserção no programa. À direita, é indicado, pela letra $x$, o número da exclusão alcançada pelos participantes e, por sombreamento, a exclusão não realizada.

A Tabela 2 apresenta a seqüência dos passos de ensino e dos passos de teste, bem como a descrição dos estímulos e das relações envolvidas.

$\mathrm{Na}$ Tabela 3 encontram-se as palavras de ensino, dissílabas ou trissílabas, compostas por sílabas simples, e as palavras de generalização, obtidas por recombinação das mesmas sílabas simples. As palavras de ensino foram escolhidas de tal maneira que uma mesma sílaba pudesse ser reforçada em mais de uma posição ( $1^{\underline{a}}, 2^{\underline{a}}$ ou $3^{\underline{a}}$ posições).

Os passos de exclusão envolveram, cada um, a introdução de três palavras de ensino (com seis tentativas da rAC, uma tentativa da $\mathrm{rAB}$ e duas tentativas das relações $\mathrm{AF}$ e $\mathrm{CF}$ para cada palavra). Para o grupo com nomeação oral, nas tentativas AF e CF, os Ps deveriam, a pedido da experimentadora, após o aparecimento do estímulo modelo, nomeá-lo oralmente e montálo durante o ensino das relações ADF (estímulo auditivo anagrama) e CDF (palavra impressa - anagrama).

Em cada passo da exclusão, a partir da Exclusão 1, as tentativas iniciais de um passo de exclusão constituíram-se num pré-teste que avaliou a leitura das palavras de ensino a serem introduzidas no passo, das palavras ensinadas na sessão imediatamente anterior (retenção) e também de três palavras de generalização, além de verificar se os Ps conheciam as figuras (rAB). As tentativas finais do passo (pós-teste) também foram usadas como sondas de leitura das palavras ensinadas e das palavras de generalização. Após o P ter atingido o critério para passar para a próxima exclusão, foi realizado um pré-teste de montagem (anagrama) das palavras de ensino, uma atividade de cópia com consequenciação positiva dos acertos e, novamente, um pós-teste de montagem (anagrama) das mesmas palavras. Passos de equivalência foram realizados depois de cada dois passos de exclusão. Ao final do programa foi realizado o teste final.

As tentativas corretas eram sempre reforçadas nos passos onde estava ocorrendo o procedimento de ensino de novas relações, através de diferentes tipos de imagens animadas e expressões verbais fornecidas pelo software Mestre@.

As imagens e as expressões verbais ocorriam em contextos em que duas crianças (um menino e uma menina) brincam. Estes feedbacks eram liberados pelo próprio programa de ensino e consistiram da apresentação na tela, após cada tarefa, da figura de uma criança jogando basquete, pulando corda, brincando de patins e brincando de skate. Quando a resposta era correta, a criança acertava a cesta, acompanhada da expressão: "muito bem!"; quando a resposta era incorreta, a criança errava a cesta, acompanhada pela expressão: "ah! você errou!". Aparecia também a figura de uma criança pulando corda; se a resposta do participante fosse correta, ela terminava a brincadeira sem cair, acompanhada da expressão "excelente!"; se o P errava, a criança caía ao pular a corda, acompanhada da expressão "oh! oh! você errou!"; a brincadeira de patins sem qualquer erro era acompanhada da expressão "perfeito!"; se este errava, aparecia a expressão "que dó, você errou!"; a brincadeira de skate com sucesso era acompanhada da expressão "é isso aí, meu!"; se este errava, aparecia a expressão “que pena, você errou!”. 
Tabela 2

Seqüência dos passos de ensino e de testes do Grupo 1 e Grupo 2, em que apenas os participantes do Grupo 1 devem nomear oralmente a palavra ditada $(r A D F)$ e impressa $(r C D F)$

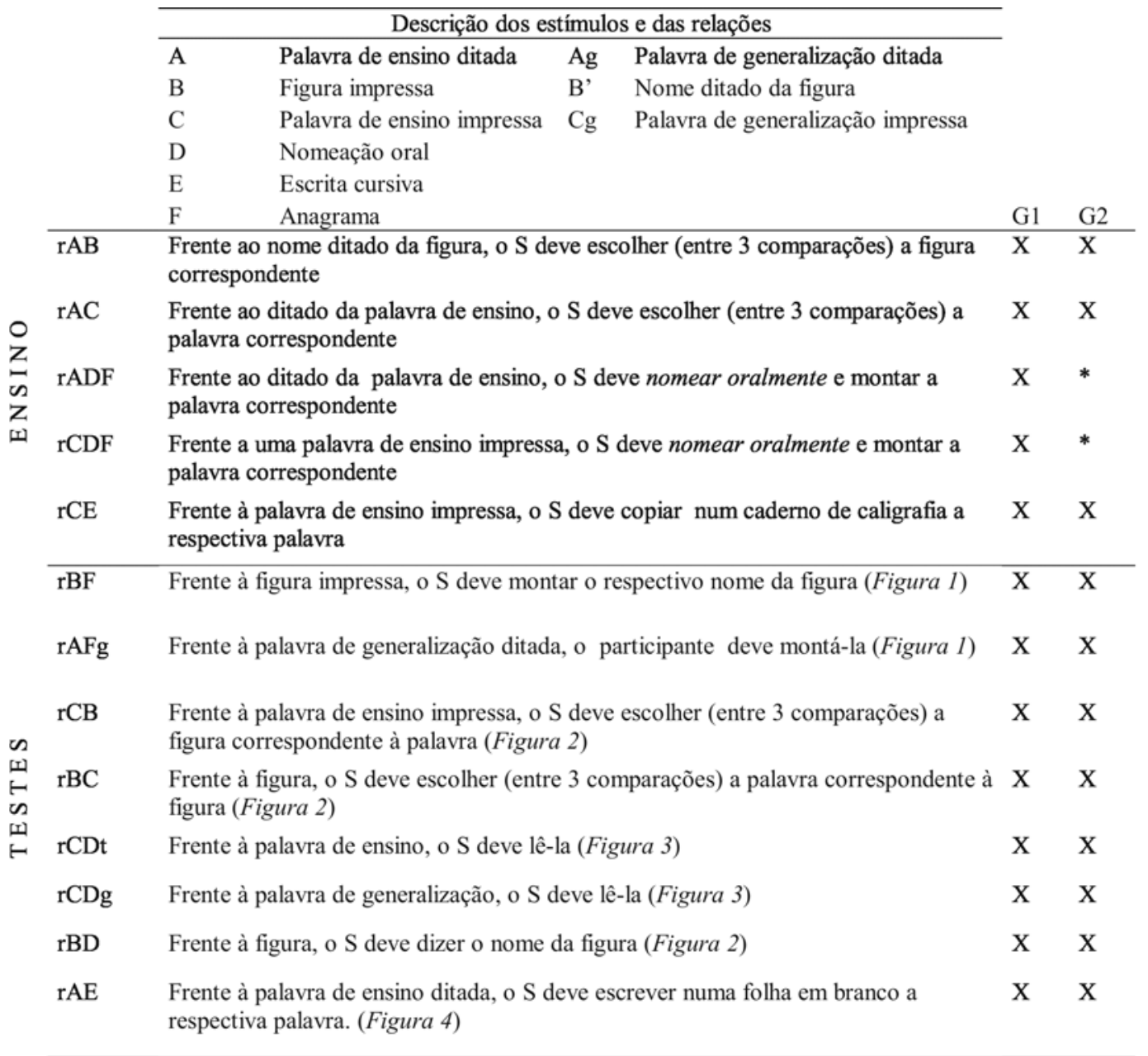

*As contingências são as mesmas, exceto que, para esse grupo, não houve nomeação oral.

Tabela 3

Relação das palavras de ensino e das palavras de generalização e posição em que as sílabas componentes das palavras de ensino foram reforçadas $\left(1^{\underline{a}}, 2^{\underline{a}}\right.$ ou $3^{\underline{a}}$ posições $)$

\begin{tabular}{|c|c|c|c|c|c|c|c|c|c|}
\hline \multirow[t]{3}{*}{ Fases } & \multicolumn{2}{|c|}{ Palavras de } & & & & & & & \\
\hline & Ensino & Generalização & \multicolumn{7}{|c|}{ Posições de reforçamento } \\
\hline & & & $1^{\underline{a}} \mathrm{p}$ & $2^{\underline{a}} \mathrm{p}$ & $1^{\underline{a}} \mathrm{p}$ & $2^{\underline{a}} \mathrm{p}$ & $1^{\underline{a}} \mathrm{p}$ & $2^{\underline{a}} \mathrm{p}$ & $3^{\underline{a}} \mathrm{p}$ \\
\hline LB & cama-mato-foca & & $\mathrm{ca}$ & $\mathrm{ca}$ & $\mathrm{ma}$ & $\mathrm{ma}$ & & & \\
\hline EXC 1 & boca-lobo-calo & toma-cato-maca & bo & bo & lo & lo & $\mathrm{ca}$ & $\mathrm{ca}$ & \\
\hline EXC 2 & bala-lago-gota & bobo-cabo-bolo & la & la & go & go & & & \\
\hline EXC 3 & doce-cela-lata & bago-bata-gola & ce & ce & la & la & & & \\
\hline EXC 4 & mico-copo-pote & tala-lado-cedo & co & co & po & po & & & \\
\hline EXC 5 & fita-taco-cola & tela-teco-comi & co & co & ta & ta & & & \\
\hline EXC 6 & macaco-camisa-caneca & fila-fico-cota & & & & & ca & $\mathrm{ca}$ & $\mathrm{ca}$ \\
\hline EXC 7 & calça-açúcar-cesta & canela-caco-saco & ce & ça & & & & & \\
\hline EXC 8 & girafa-barraca-colar & cear-alça-caçar & & $\mathrm{ra}$ & & rra & & & \\
\hline EXC 9 & ninho-minhoca-lenha & garrafa-corra-girar & & nho & & nha & & & \\
\hline EXC 10 & anjo-bandeira-balança & caminho-fofinha-tenho & an & an & & & & & \\
\hline EXC 11 & palhaço-abelha-velho & lança-anta-banco & & lha & & lho & & & \\
\hline EXC 12 & queijo-coqueiro-leque & palha-olho-folha & quei & quei & & que & & & \\
\hline EXC 13 & soldado-pastel-pulseira & pequeno-moleque-quero & pa & & $\mathrm{pu}$ & & & & \\
\hline
\end{tabular}


O critério para avançar para um passo seguinte foi: (a) Pré-Teste: $100 \%$ de acerto, em uma sessão, da leitura das palavras da Linha de Base. Se o $\mathrm{P}$ não respondesse neste nível de acerto, o E apresentava a Exclusão imediatamente anterior; (b) Exclusão: 91,6\% de acerto em uma sessão, entre a montagem nas relações AF (estímulo auditivo - montagem) e a montagem nas relações CF (palavra impressa - montagem); 95,2\% entre a identificação das relações $A B$ (estímulo auditivo - figura), e das relações AC (estímulo auditivo palavra impressa) e (c) Pós-Teste: 100\% de acerto em uma sessão na relação AC das palavras de ensino.

\section{Resultados e Discussão}

\section{Relação AFg}

A Figura 1 apresenta, na parte superior, o resultado do teste de montagem das palavras de generalização (rAFg) frente ao estímulo auditivo. Pode ser observado que, dos seis Ps do grupo com nomeação oral, dois montaram as palavras de generalização (P3 e P11) com percentual de acertos entre 80 e $100 \%$ e um (P1) realizou apenas $50 \%$ das montagens. P2 e P4 que realizaram as tarefas até a exclusão 7 e P5 que não foi além da exclusão 1, não conseguiram montar as palavras, aparecendo com desempenho próximo de zero.

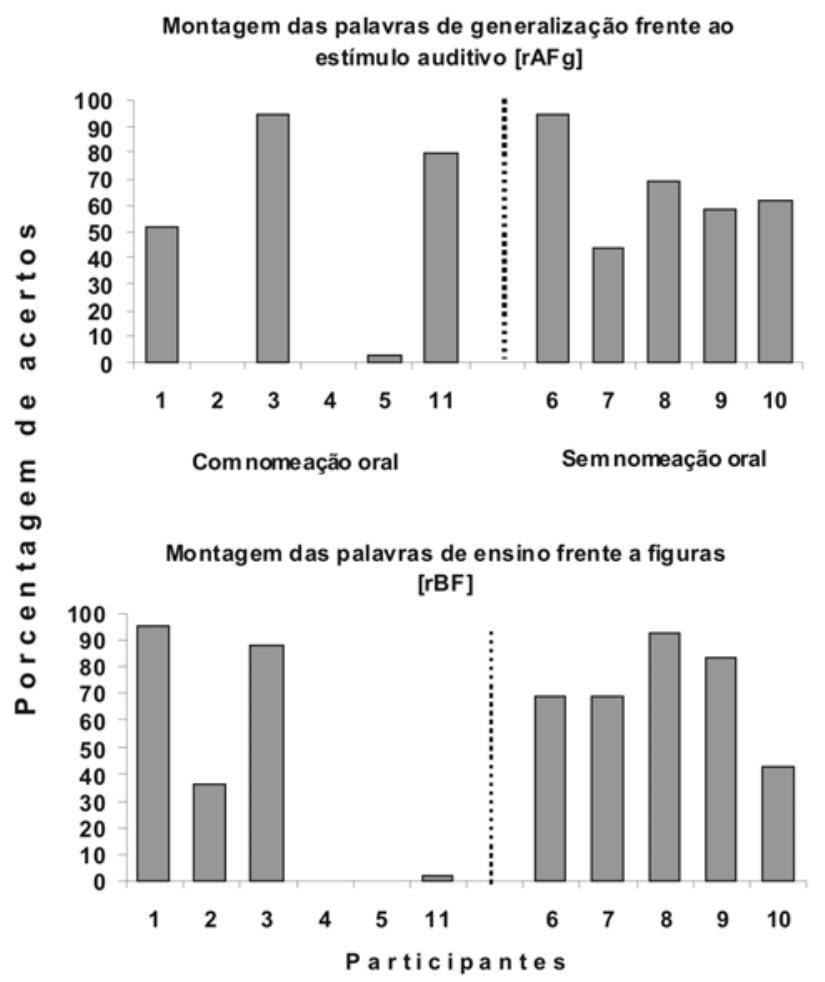

Figura 1. Porcentagem de acertos nos testes de montagem das palavras de generalização [rAFg (parte superior)] frente ao estímulo auditivo e de montagem das palavras de ensino frente a figuras [rBF (parte inferior)].
Todos os Ps do grupo sem nomeação oral realizaram as montagens, em que P6 aparece com o melhor desempenho $(94,8 \%)$; os Ps 8,9 e 10 realizaram em torno de $65 \%$ e P7 em torno de $50 \%$ das tarefas de montagem.

\section{Relação $B F$}

Encontra-se, na parte inferior da Figura 1, o resultado do teste de montagem das palavras de ensino frente às figuras (rBF). Do grupo com nomeação oral, apenas três realizaram as tarefas, em que P1 e P3 apresentam tarefas de montagem acima de $85 \%$, e P2 em torno de $35 \%$. Do grupo sem nomeação oral, todos realizaram as tarefas com desempenho acima de $70 \%$, exceto P10, cujo desempenho permaneceu próximo de $50 \%$.

\section{Relação BD}

Na parte superior da Figura 2, encontram-se os testes de identificação do nome das figuras (rBD). Neste teste praticamente todos os Ps conseguiram identificar os nomes das figuras corretamente (acima de 95\% de acerto), com exceção de P2, com $76,2 \%$ e P5, com $85,7 \%$. Pode-se observar, também, que não há diferença entre os grupos com e sem nomeação oral.

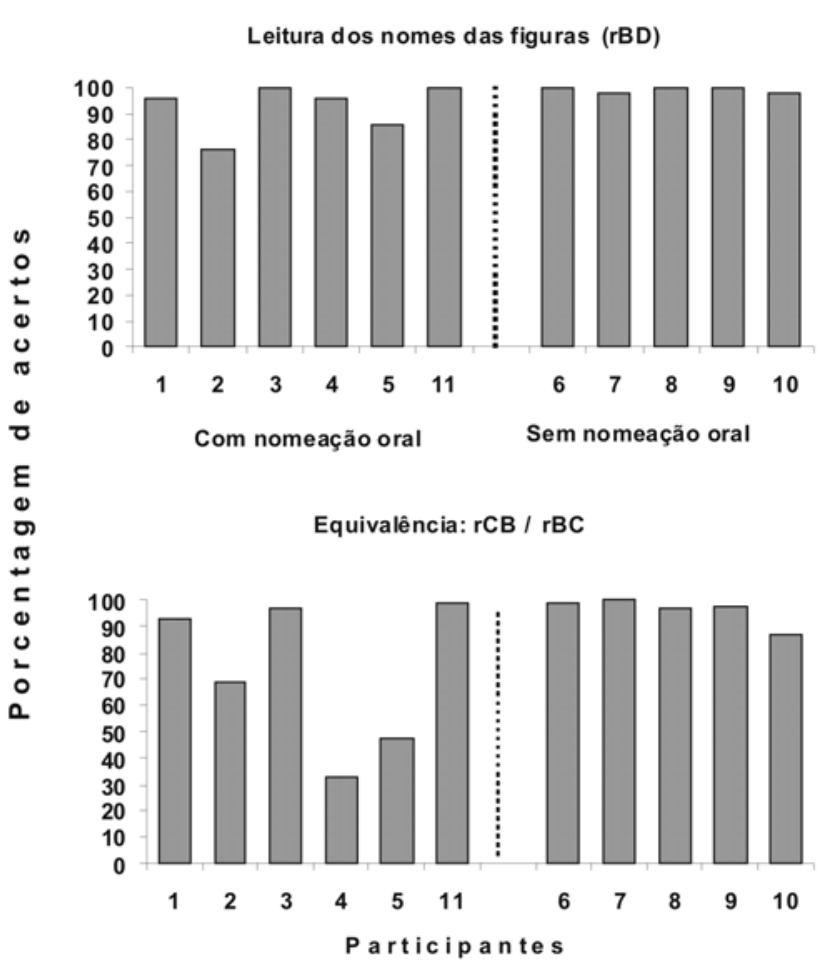

Figura 2. Identificação dos nomes das figuras frente às figuras [rBD (parte superior)] e relações de equivalência entre figuras e seus nomes $(\mathrm{rBC})$ e entre seus nomes e figuras (rCB). 


\section{Relação $B C / C B$}

Na parte inferior da Figura 2 encontram-se os resultados do desempenho em equivalência ( $\mathrm{rBC}$ e $\mathrm{rCB}$ ). No grupo com nomeação oral, P1, P3 e P11 realizaram a tarefa com aproveitamento superior a $92,8 \%$. Os demais Ps do mesmo grupo apresentam desempenho com porcentagem inferior: P2 com $70 \%$ e $\mathrm{P} 4$ e $\mathrm{P} 5$ com desempenho em torno de $40 \%$ de acertos. No grupo sem nomeação oral, o percentual de acertos situase acima de $85 \%$ para todos os Ps.

\section{Relação $C D g$ e $C D t$}

Na Figura 3, encontram-se os resultados dos testes de leitura (das palavras de generalização $[\mathrm{rCDg}]$ e das palavras de ensino [rCDt]). No grupo com nomeação oral, P1, P3 e P11 leram, praticamente, todas as palavras de generalização apresentadas, porém os Ps 2, 4 e 5, que não passaram da exclusão 7, apresentaram desempenho inferior a $3 \%$. No grupo sem nomeação oral, todos os Ps apresentam desempenho entre 90 e $100 \%$.

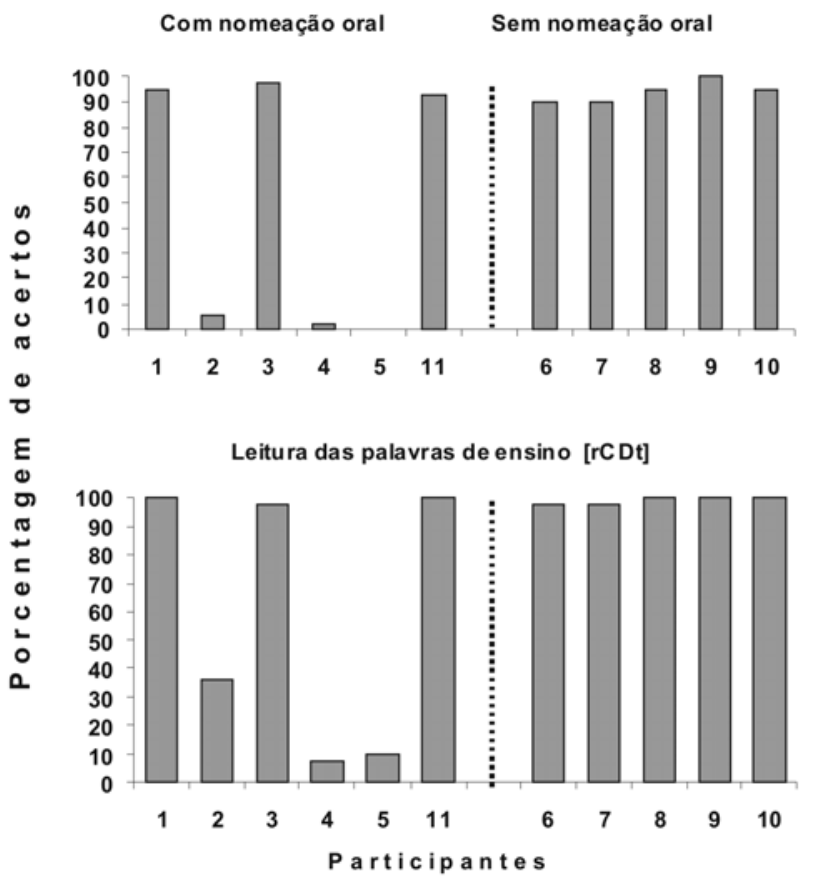

Figura 3. Porcentagem de acertos nos testes de leitura das palavras de generalização $[\mathrm{rCDg}$ (na parte superior da figura)] e das palavras de ensino [rCDt (na parte inferior da figura)].

Na parte inferior da Figura 3, pode-se observar o desempenho dos Ps na leitura de palavras de ensino. No grupo com nomeação oral, P1, P3 e P11 leram, praticamente, todas as palavras de ensino apresentadas, porém os Ps 2,4 e 5 , que não passaram da exclusão 7 , apresentam desempenho inferior: $\mathrm{P} 2$ com $35,7 \%$ e P4 e P5 com desempenho em torno de $10 \%$ de acertos. No grupo sem nomeação oral, todos os Ps apresentam desempenho entre 97 e 100\% de acertos.

A comparação entre os dois grupos de Ps (com e sem nomeação oral) ficou prejudicada pelo fato de três dos seis Ps (P2, P4 e P5) não terem ultrapassado a exclusão 7 durante a etapa de ensino das relações. Mesmo assim, pode-se notar, em todas as relações testadas ( $\mathrm{AFg}, \mathrm{BF}, \mathrm{BD}, \mathrm{CB} / \mathrm{BC}, \mathrm{CDg}$ e $\mathrm{CDt}$ ), superioridade em termos de desempenho para os Ps que não tiveram que nomear os estímulos. Além disso, a variabilidade comportamental é também menos acentuada para os Ps do grupo sem nomeação oral.

\section{Ditado pré e pós prática de caligrafia}

A Figura 4 apresenta a porcentagem de palavras escritas e classificadas dentro de classes percentuais de acertos de 0$33 \%, 50-67 \%$ e $90-100 \%$. Na parte superior encontram-se as palavras escritas na classe de $0-33 \%$ de acertos. Pode-se observar que há uma maior porcentagem de palavras concentradas nesta classe para os Ps do grupo com nomeação do que para os Ps do grupo sem nomeação.

Na parte intermediária da Figura 4, encontram-se as palavras escritas na classe de $50-67 \%$ de acertos. Os dados entre os grupos com e sem nomeação oral são, nesta classe, bastante semelhantes, com uma porcentagem um pouco superior para o grupo com nomeação.

$\mathrm{Na}$ parte inferior, encontram-se as palavras escritas na classe de $90-100 \%$ de acertos. Por ser visto, nesta classe de acertos, que a porcentagem mais elevada pertence ao grupo sem nomeação. No caso da distribuição de porcentagem de acertos dentro das classes, pode ser visto que, no grupo com nomeação, ela é bastante irregular e variável. Ao contrário, para o grupo sem nomeação, a distribuição de acertos é crescente (poucos acertos na classe de 0-33\%, quantidade média de acertos na classe de 50-67\% e maior número de acertos na classe de 90-100\%), além de mostrar pouca variabilidade.

Os dados obtidos com o teste de ditado parecem indicar que os Ps aprenderam, por generalização, a "realizar ditado" sem terem sido especificamente ensinados a fazer isso. Ou seja, foram ensinados a escolher (apontar com o mouse) uma palavra escrita na presença do som desta palavra (rAC) e, no teste de ditado (rAE), a escrever cursivamente uma palavra ditada pela experimentadora. Contudo entre o pré-teste e o pós-teste havia uma atividade de cópia da palavra que estava sendo ditada reforçada positivamente. Os dados indicam que a atividade de cópia não produziu efeito significativo entre o pré e o pós-teste, pois a diferença percentual entre estas etapas é muito pequena.

A Figura 5 apresenta os dados organizados por grupo. Comparando-se o desempenho no pós-teste destes grupos, pode-se perceber uma percentual maior de palavras para o grupo com nomeação $(14,4$ versus 2,3$)$ na classe de acertos de 0 $33 \%$. Na classe de acertos de 50-67\% a diferença entre os grupos diminui, porém com valor mais alto $(14,4$ versus 9,9$)$ no pós-teste para o grupo com nomeação. Na classe de acertos de $90-100 \%$, o resultado se inverte, ou seja, o grupo sem nomeação apresenta um desempenho superior $(87,7$ versus 71,3$)$. 


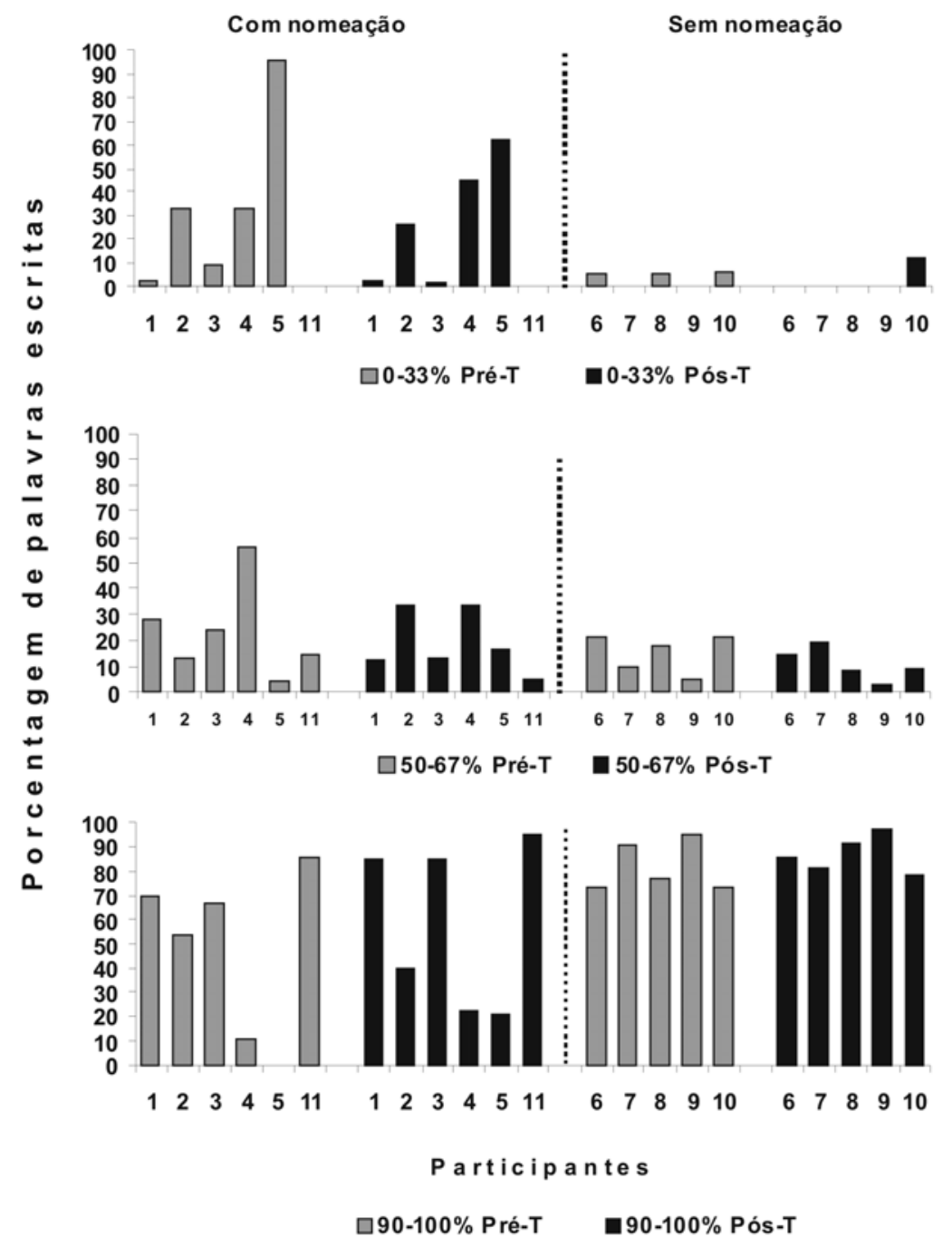

Figura 4. Porcentagem de palavras de ensino escritas pelo participante nos pré e pós-testes de ditado com treino de caligrafia entre eles, dentro das classes de acertos de $0-33 \%, 50-67 \%$ e $90-100 \%$, bem como as respectivas medianas por etapas (pré, pós) e por condição (com e sem nomeação).

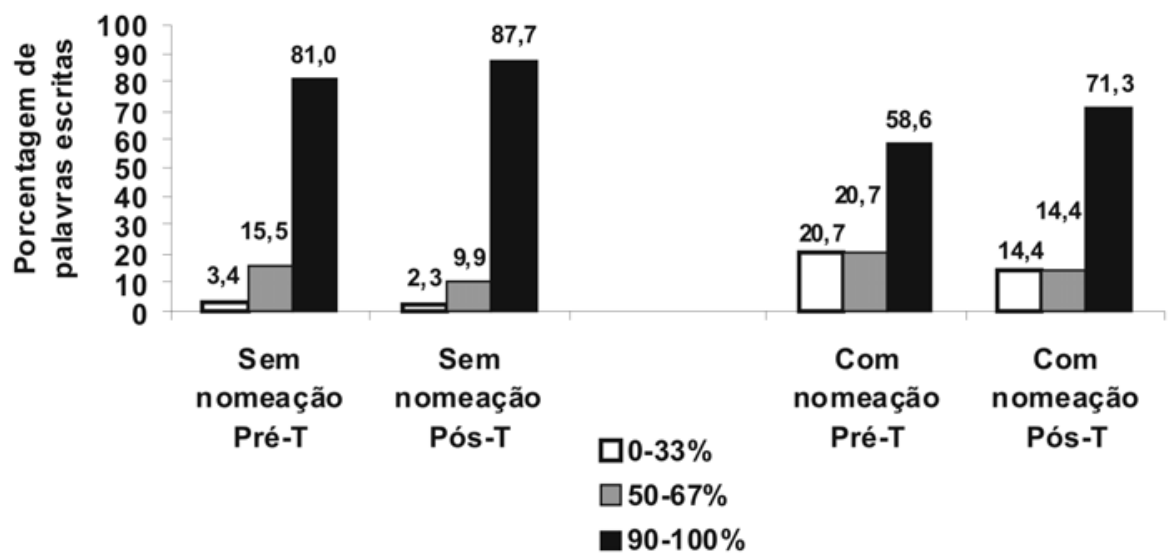

Figura 5. Porcentagem de palavras de ensino escritas, agrupadas por condição, nas etapas dos pré e pós-testes de ditado com treino de caligrafia entre eles, dentro das classes de acertos de $0-33 \%$, 50-67\% e $90-100 \%$. 


\section{Posição das sílabas no treino}

Uma das condições que pode aumentar a probabilidade de leitura das palavras de generalização é o reforçamento da posição das sílabas durante a etapa de ensino das palavras. No presente procedimento as sílabas foram alocadas em diferentes posições como pode ser visto na Tabela 3. Por exemplo, nas palavras caMA e MAto, o MA foi reforçado tanto na segunda como na primeira posição e nas palavras CAma e foCA, o CA foi reforçado tanto na primeira como na segunda posição. Organização semelhante ocorreu com palavras de ensino até a exclusão 5. Desta em diante, com o uso de palavras trissílabas, a repetição (e conseqüente reforçamento) das mesmas sílabas em diferentes posições não ocorreu pela dificuldade de encontrar palavras com esse tipo de formação. $\mathrm{Na}$ organização das palavras de generalização, estas foram montadas com as sílabas das palavras de ensino. Como, na etapa de ensino, elas foram reforçadas em diferentes posições, é provável que a alta porcentagem de leitura correta das palavras de generalização possa ser explicada pelo uso dessa estratégia, conforme defendido por Matos e colaboradores (1997).

\section{Efeitos da nomeação oral}

Há controvérsias quanto aos efeitos da nomeação oral sobre a formação de classes de equivalência. Alguns autores (Sidman, 1986, 1990, 1994; Sidman et al., 1982; 1986) não vêem necessidade de se nomear oralmente os estímulos para que haja formação dessas classes. Outros, como Devany e colaboradores (1986), Dugdale e Lowe (1990), Monteiro e Medeiros (2002), argumentam que tal procedimento pode ser uma forma efetiva de facilitar a emergência de classes equivalentes.

No procedimento realizado observou-se efeito diferencial a favor do grupo sem nomeação, contrariando, portanto, resultados obtidos por Monteiro e Medeiros (2002) para o desempenho matemático e em concordância com autores como Sidman (1986, 1990, 1994) e Sidman e colaboradores (1982, 1986), que não vêem necessidade de se nomear oralmente os estímulos para que haja formação dessas classes. Os Ps 2, 4 e 5 não mostram desempenho superior, mesmo nomeando oralmente os estímulos, além de não terem passado da exclusão 7. Verificou-se ainda que, de maneira geral, os Ps que não realizaram o procedimento de nomeação oral apresentam melhor desempenho, com exceção de P7, que na montagem das palavras de generalização frente ao estímulo auditivo também não ultrapassou a exclusão 7 . Era esperado, segundo a proposição dos autores que defendem a facilitação pela nomeação oral, que o procedimento facilitasse o desempenho de todos os Ps, contudo não foi o que ocorreu, principalmente com P2, P4 e P5.

Concluindo, pode-se dizer que as relações ensinadas e testadas demonstram que um programa computadorizado é factível de ser usado com crianças de uma escola da rede pública que apresentam dificuldades de aprendizagem. Os computadores dos laboratórios de informática das escolas da rede pública têm sido utilizados de maneira parcial, isto é, têm servido basicamente para acessar a Internet e para a rea- lização de tarefas escolares. A utilização do software Mestre@ para ensinar leitura e escrita a crianças com dificuldades de aprendizagem mostrou que o computador pode ser usado para funções mais significativas.

Em relação ao desempenho acadêmico em sala de aula, os Ps 5 e 7, que não passaram da exclusão 7, não obtiveram nota suficiente para serem aprovados, o mesmo ocorrendo com P6, mesmo tendo completado todas as exclusões. O restante dos alunos alcançou a média necessária para passar de ano.

$\mathrm{O}$ desempenho de $\mathrm{P} 4$ pode ter sido influenciado por uma aparente falta de motivação para a realização das tarefas no computador pois, em alguns momentos, ele se mostrava "deprimido". No procedimento, P4 não foi além da exclusão 1 , ou seja, não conseguiu avançar no procedimento, como os demais Ps. Levantou-se, também, a possibilidade do fato do pai de P4 ter sido diagnosticado com câncer e estar sendo submetido à quimioterapia ter influenciado seu desempenho no procedimento. Ele não conseguiu passar da tarefa 1, tendo sérias dificuldades de leitura e escrita (durante os ditados). Em sala de aula, a professora queixou-se muito do não cumprimento das atividades por parte deste participante.

O desempenho de P5 também não foi satisfatório, apesar de ter alcançado a exclusão 7. Seu desempenho nas atividades mostrou uma melhora significativa no segundo semestre do ano. Durante o ano a professora queixou-se de que P5 mostrava desinteresse pelas aulas e que "fazia muita bagunça em sala". Esses casos requerem um procedimento de coleta de dados que vai além do procedimento experimental. Seria necessário observá-los em outros contextos (por exemplo, sala de aula, recreação, residência, etc.) para que se ter, com mais clareza, um quadro das relações entre variáveis.

\section{Referências}

de Rose, J. C. C. (1988). Equivalência de estímulos: problemas atuais de pesquisa [Anais]. In Sociedade Brasileira de Psicologia (Org.), Anais da Reunião Anual de Psicologia (p. 19-32). Ribeirão Preto: Autor.

de Rose, J. C. C., Souza, D. G., Rossito, A. L., \& de Rose, T. M. S. (1989). Equivalência de estímulos e generalização na aquisição da leitura após história de fracasso escolar. Psicologia: Teoria e Pesquisa, 5, 325-346.

Devany, J. M., Hayes, S. C., \& Nelson, R. O. (1986). Equivalence class formation in language-able and language-disabled children. Journal of the Experimental Analysis of Behavior, 27, 433-442.

Dugdale, N., \& Lowe, C. F. (1990). Naming and stimulus equivalence. In D. E. Blackman \& H. Lejeune (Orgs.), Behaviour analysis in theory and practice: contributions and controversies (pp. 115-138). Brighton, Reino Unido: Earlbaum.

Gatti, B. A. (1993). Os agentes escolares e o computador no ensino. Revista de Educação e Informática, $\mathrm{n}^{\mathrm{o}}$ especial, 22-27.

Goyos, C., \& Almeida, J. C. (1996). Mestre (Versão 1.0) [Programa de computador]. São Carlos, SP: Mestre Software.

Hanna, E. S., Souza, D.G., de Rose, J. C., Santos, L. G., Fonseca, M. L., Horowitz, D. B., Carvalho, G. P., Sallorenzo, L. H., Balduíno, L. H., \& Veiga, H. (1993). Treino de cópia com resposta construída e o desempenho em ditado II: efeitos dependentes do nível de generalização na linha de base de leitura [Resumo]. In Sociedade Brasileira de Psicologia (Org.), XXIII Reunião Anual de Psicologia (p. 305). Ribeirão Preto: Autor.

Hayes, S. C. (1989). Nonhumans have not yet shown stimulus equivalence. Journal of the Experimental Analysis of Behavior, 51, 385-392. 
Mackay, H. A. (1985). Stimulus equivalence in rudimentary reading and spelling. Analisys and Intervention in Developmental Disabilities, 5, 373-387.

Mackay, H.A., \& Sidman, M. (1984). Teaching new behavior via equivalence relations. In P. H. Brooks, R. Sperber, \& C. McCauley (Orgs.), Learning and cognition in the mentally retarded (pp. 493-513). Hillsdale, New Jersey: Erlbaum.

Matos, M. A., Peres, W., Hübner, M. M., \& Malheiros, R. H. S. (1997). Oralização e cópia: efeitos sobre a aquisição de leitura generalizada recombinativa. Temas em Psicologia, 1, 47-64.

McDonagh, E. C., McIlvane, W. J., \& Stoddard-Lawrence, T. (1984). Teaching coin equivalences via matching to sample. Applied Research in Mental Retardation, 5, 177-197.

Medeiros, J. G. (1997). Relato de uma experiência de ensinar: construindo a relação entre teoria e prática. Temas em Psicologia, 1, 7-22.

Medeiros, J. G., Antonakopoulu, A., Amorim, K., \& Righetto, A. C. (1997). O uso da discriminação condicional no ensino da leitura e escrita. Temas em Psicologia, 1, 23-32.

Medeiros, J. G., Baus, J., Jeremias, A. E., Mattos, V., Freitas, A. N., Sengl, C. S., Silva, I. W., Silva, M. H., Monteiro, C. R., Dutra, G., \& Franco, R. (1995). A utilização do procedimento de discriminação condicional como estratégia para a consecução de objetivos de ensino [Resumo]. In Sociedade Brasileira de Psicologia (Org.), XXV Reunião Anual de Psicologia (p. 515). Ribeirão Preto: Autor.

Medeiros, J. G., Konescki, G. E., \& Souza, A. P. (1993). Alternativa de alfabetização para crianças com dificuldades de aprendizagem em leitura e escrita [Resumo]. In Departamento de Apoio à Pesquisa UFSC (Org.), III Seminário Catarinense de Iniciação Científica (p. 50). Florianópolis: Autor.

Medeiros, J. G., \& Monteiro, C. R. (1996). Modificação de comportamento de uma criança com distúrbio de déficit de atenção com hiperatividade, com a participação dos familiares: um estudo de caso [Resumo]. In Sociedade Brasileira para o Progresso da Ciência (Org.), III Reunião Especial da SBPC (p. 527). Florianópolis: Autor.

Medeiros, J. G., Monteiro, G., \& Zaccaron, K. (1997). Aquisição de leitura e escrita a um sujeito adulto. Temas em Psicologia, 1, 65-78.

Medeiros, J. G., \& Silva, R. M. F. (2002). Efeitos de testes de leitura sobre a generalização em crianças em processo de alfabetização. Psicologia: Reflexão e Crítica, 15, 587-602.

Medeiros, J. G., \& Teixeira, S. A. (2000). Ensino de leitura e escrita através do pareamento com o modelo e seus efeitos sobre medidas de inteligência. Estudos de Psicologia, 5, 181-214.

Medeiros, J. G., Teixeira, C. M., Cabral, C., Silva, A. B. C., \& Brandão, L. (1995). Observação e registro da interação professor-aluno junto a uma classe com dificuldades de aprendizagem [Resumo]. In Sociedade Interamericana de Psicologia (Org.), XXV Congresso Interamericano de Psicologia. San Juan, Porto Rico: Autor.
Melchiori, L., Souza, D., \& de Rose, J. (1992). Aprendizagem de leitura por meio de um procedimento de discriminação sem erros (exclusão): uma replicação com pré-escolares. Psicologia: Teoria e Pesquisa, 8, 101-111.

Melchiori, L., Souza, D., de Rose, J., \& Furlan, C. (1992). Aquisição de leitura por adultos analfabetos, após ensino de discriminações condicionais entre palavras impressas e palavras ditadas [Resumo]. In Sociedade Brasileira de Psicologia (Org.), XXV Reunião Anual de Psicologia (p. 92). Ribeirão Preto: Autor.

Monteiro, G., \& Medeiros, J. G. (2002). A contagem oral como pré-requisito para a aquisição do conceito de número com crianças pré-escolares. Estudos de Psicologia, 7, 73-90.

Ribeiro, M. J. L. (1997). Dificuldades de leitura: capacitação de professores para a utilização de uma metodologia de ensino informatizada. Dissertação de Mestrado não-publicada, Universidade Estadual de Londrina, Londrina.

Rodrigues, V., \& Medeiros, J. G. (2001). A utilização da discriminação condicional no ensino da leitura e escrita a crianças com paralisia cerebral. Estudos de Psicologia, 18, 55-73.

Sidman, M. (1986). Functional analysis of emergent verbal classes. In T. Thompson \& M. Zeiler (Orgs.), Analysis and integration of behavioral units (pp. 213245). Hillsdale, New Jersey: Erlbaum.

Sidman, M. (1990). Equivalence relations: where do they come from? In D. E. Blackman \& H. Lejeune (Orgs.), Behaviour analysis in theory and practice: contributions and controversies (pp. 93-114). Brighton, Reino Unido: Earlbaum.

Sidman, M. (1994). Equivalence relations and behavior: a research story. Boston: Authors Cooperative.

Sidman, M., Rauzin, R., Lazar, R., Cunningham, S., Tailby, W., \& Carrigan, P. (1982). A search for symmetry in the conditional discriminations of rhesus monkeys, baboons, and children. Journal of the Experimental Analysis of Behavior, 37, 23-44.

Sidman, M., Wilson-Morris, M., \& Kirk, B. (1986). Matching-to-sample procedures and the development of equivalence relations: the role of naming. Analysis and Intervention in Developmental Disabilities, 6, 1-19.

Singh, N. N., \& Singh, J. (1986). Reading acquisition and remediation in the mentally retarded. In N. R. Ellis \& N. W. Bray (Orgs.), International Review of Research on Mental Retardation (pp. 165-199). Nova York: Academic.

Stromer, R. (1991). Stimulus equivalence: implications for teaching. In W. Ishaq (Org), Human behavior in today's world (pp. 109-122). Nova York: Praeger.

Strommer, R., \& Mackay, A. H. (1992). Delayed constructed-response identify matching improves the spelling performances of students with mental retardation. Journal of Behavioral Education, 2, 139-158.

Strommer, R., Mackay, A. H., \& Stoddard, L. T. (1992). Classroom applications of stimulus equivalence technology. Journal of Behavioral Education, 2, 225-256.

José Gonçalves Medeiros, doutor em Psicologia Experimental pela Universidade de São Paulo, é professor no departamento de Psicologia da Universidade Federal de Santa Catarina, bolsista pesquisador do CNPq e coordenador do Laboratório de Linguagem e Comportamento Verbal. Endereço para correspondência: Caixa Postal 5060; Florianópolis, SC; CEP 88040-970. Fones: (48) 9972-4042 / 237-4283 / 331-8565 / 331- 9457. E-mail: medeiros@mbox1.ufsc.br Analu Regis Fernandes é bolsista PIBIC e aluna do curso de graduação em Psicologia da Universidade Federal de Santa Catarina.

Raquel Pimentel é bolsista PIBIC e aluna do curso de graduação em Psicologia da Universidade Federal de Santa Catarina.

Ana Carolina Seara Simone é bolsista de IC/CNPq e aluna do curso de graduação em Psicologia da Universidade Federal de Santa Catarina. 